\title{
Human opinion dynamics-based optimisation for in-band spectrum sensing in cognitive radio networks
}

\author{
Roopali Garg ${ }^{1 *}$ and Nitin Saluja ${ }^{2}$ \\ ${ }^{1}$ University Institute of Engineering and Technology, Punjab University, Chandigarh, India. \\ ${ }^{2}$ Chitkara University Institute of Engineering and Technology, Chitkara University, Panjab, India.
}

\begin{abstract}
In cognitive radios, the primary user sensing plays an important role in defining a compromise between spectrum efficiency and licensed user interference. In this paper, the performance of cognitive radio is optimised using human opinion dynamics (HOD) inspired continuous opinion dynamics optimiser (CODO) with sensing parameters and throughput as arguments of objective function. The optimisation parameters are throughput, probability of false alarm and sensing time under varying signal to noise ratio (SNR) conditions with fixed frame period and at least $90 \%$ probability of detection. Further, a new concept of human interaction for forming opinions is proposed to explore the idea of sensing-throughput optimisation. A statistical analysis showed that the proposed algorithm exhibits an improvement of $0.2 \%$ in probability of detection with the condition that probability of detection is at least $90 \%$, as per the Federal Communication Commission (FCC) guidelines. Here, the frame period is kept fixed at $224.25 \mathrm{~ms}$ and the sensing time does not exceed $31.59 \mathrm{~ms}$. There is also a drop of $5.25 \%$ in false reports and an enhancement of $0.08 \%$ in normalised throughput as compared to swarm intelligence technique when probability of detection is $95 \%$ for the above frame period and sensing time. This implies that the cognitive user will not have to vacate the band on wrong reporting and can continue transmitting the data until the primary signal is indeed detected. Further, a maximum normalised throughput of 0.9088 is recorded at a minimum sensing time of $17.10 \mathrm{~ms}$ indicating that only $\sim 8 \%$ of the frame is utilised for sensing.
\end{abstract}

Keywords: Cognitive radio, human opinion dynamics, primary user, region of convergence, secondary user.

\section{INTRODUCTION}

Cognitive radio (CR) is an ideation from Joseph Mitola III (Giupponi et al., 2010). CRs are intelligent devices that have the capability to sense radio environment. In the cognitive environment, users are classified as either primary user (PU) or secondary user (SU). PU is a licensed user having higher priority over the SU who seeks free channel for sharing. Hence, the SUs should have capabilities to sense the neighbour channels for availability of unused bands called spectrum holes or white spaces. The spectrum hole is selected to optimise the system parameters and the selection depends on the channel statistics (caused due to interference, random fading, PU activities etc.), channel estimations (like noise variance, SNR estimate etc.), Bit error rate (BER), quality of service (QoS), data transmission rate, etc. SU starts transmitting data in the sensed spectrum hole; and if PU is detected then SU releases the spectrum and looks for the next spectrum hole available (Garg \& Saluja, 2016).

The literature is rich in the domain of spectrum awareness and spectrum exploitation of cognitive radio communication (Yücek \& Arslan, 2009). Ozbay \& Ercelebi (2015) proposed a novel spectrum sensing detection scheme to detect PU considering the additive white Gaussian noise (AWGN) channel. Furthermore, the

\footnotetext{
* Corresponding author (roopali.garg@gmail.com; (iD https://orcid.org/0000-0003-0508-5149)
} 
paper compares different detectors, viz. energy detector, cooperative energy detector, Anderson-Darlington and Kolomogorov-Smirnov detector. The performance parameters considered are probability of false alarm and (SNR). Further, the decision in context of cooperative spectrum sensing is done by utilising Kernel Least Mean Square (KLMS) algorithm in $\mathrm{Xu}$ et al. (2015). The authors analyse mean square error calculated for varying SNR conditions and number of users. It is also used in defining parameters of system for analysis. Similarly, the probability of false alarm is derived in (So \& Kwon, 2016) in addition to the average capacity of SUs, which is claimed to be affected by sensing time, reporting time and fusion scheme. Furthermore, the optimisation of sensing-throughput compromise has been addressed by considering the whole frame period in (Stotas \& Nallanthan, 2010). The capacity of SU is evaluated when there is limited knowledge of the channel in Smith et al. (2012). The probability of detection with energy detection sensing scheme and non-fading AWGN channel for varying SNR conditions is considered (Pandya et al., 2015) and followed by comparison of energy detector and feature detector (Kim \& Shin, 2008). It is claimed that the energy detection scheme incurred less sensing overhead for the varying SNR values. In Liang et al. (2008), an optimal trade-off between throughput and probability of false alarm is derived. A suitable sensing time is chosen with an assumption of periodic sensing. Aulakh \& Vig (2015) have made an attempt to maximise the secondary user access by optimising its sensing time with respect to the collision cost in a thresholdbased sensing-transmission structure. An optimisation algorithm is suggested in Liu et al., (2015) to maximise throughput and to optimise sensing time with respect to number of users for cooperative sensing with low time complexity using OR rule. Xiao et al. (2014) have utilised the concept of wideband spectrum sensing for cognitive radio networks to improve the throughput. Here, the spectrum is considered to comprise of several narrow bands. The sensing information of all these narrow bands is aggregated to make a final decision for the wideband spectrum. The design utilises power allocation strategy to optimise the sensing time, improve the throughput, and save power consumption. Zhang et al. (2016) explored the sensing-throughput trade-off problem by taking the impact of imperfect spectrum sensing and multichannel access contention into consideration. Here, the constraint is imposed on interference probability instead of probability of detection. The application of machine learning is extensively applied in the field of cognitive radios (Clancy et al., 2007; Bkassiny et al., 2013;
Thilina et al., 2013). Particle swarm optimisation (PSO) and ant colony optimisation (ACO) are two popular swarm intelligence techniques applied to varied domains like tuning of GPS-aided attitude estimation (Poddar et al., 2016), modelling particle behaviour to assess the premature convergence (Wickramage \& Ranasinghe, 2014), etc. Rashid et al. (2015) have utilised a variant of PSO technique (i.e. fast convergent PSO) to address the issue of sensing-throughput by defining a stopping threshold. Hybrid of PSO and ACO is implemented (Jhajj et al., 2017) to check the efficacy for spectrum sensing in cognitive radios. Suseela \& Sivakumar, (2017) utilises a multichannel-based tree-seed algorithm to have low probability of false alarm and higher throughput without compromising transmission rate. The convergence time is found to be faster in this case.

Dempster-Shafer theory (DST) is a concept of sensor fusion, which combines the degree of belief. This combining technique minimises the uncertainty and makes a final decision by focusing on certain important information (Wickramarathne et al., 2013). Human opinion models are based on the social behaviour of intelligent human beings. The decision of each being is taken into consideration in making the final decision. There have been several human opinion models suggested by researchers. Social impact theory-based optimiser (SITO) is based on opinion formation for solving optimisation problems (Macaš et al., 2012). This optimization algorithm is based on discrete opinion and is restricted to small dimensional real valued problems. Deffuant et al. (2000) and Hegselmann and Krause, (2005) proposed a continuous opinion-based model. They assumed a preset value for interaction amongst individuals. The final solution is always well defined and led to fast convergence of opinion. An 'opinion noise', which causes a large and unexpected change in opinion of the individuals, is added to avoid premature convergence. Computational model in Mäs et al. (2010) led to development of another model called continuous opinion dynamics optimizer (CODO) (Kaur et al., 2013).

\section{METHODOLOGY}

There are two stages of spectrum sensing: wideband sensing followed by in-band sensing. Wideband sensing involves the prediction of length of channel available based on the channel-state-information algorithms. The spectrum holes are primarily detected in this phase. In in-band sensing or fine sensing, the interest is to predict $\mathrm{PU}$ to minimise the interference. 
Spectrum sensing model considers two global hypotheses:

Global hypothesis 1: The frame period $\left(T_{f}\right)$ is constant and is given by,

$$
T_{f}=T_{s}+T_{d}
$$

Where, $T_{s}$ is sensing time i.e. time taken to sense the spectrum. $T_{d}$ is data transmission time i.e. time taken to transmit the data. Greater transmit time ensures more throughput. $T_{f}$ is frame period, which is sum of sensing time and data transmission time. It implies that the sensing frequency is $\frac{1}{T_{f}}$.

Global hypothesis 2: Longer sensing time results in greater probability of detection $\left(\mathrm{P}_{\mathrm{d}}\right)$ and lower probability of false alarm $\left(\mathrm{P}_{\mathrm{f}}\right)$, but it leads to reduced data transmission time as the frame period is fixed and hence results in reduced throughput.

\section{Problem formulation}

A large sensing time is desirable to have good primary signal detection, but it leads to reduced data transmission time. It is postulated to have trade-off between sensing time and throughput and the same is targeted to optimise in this paper. It is subject to the condition that the interest of PU is sufficiently protected. Spectrum sensing can be formulated with two hypotheses:

$H_{0}$ : Channel is temporarily free i.e. PU is absent

$H_{1}$ : Channel is occupied i.e. PU is present

The spectrum-sensing problem is to decide between null hypothesis $H_{0}$ and alternate hypothesis $H_{1}$ :

Null hypothesis:

$$
H_{0}: S[n]=U[n]: P U \text { is absent }
$$

Alternate hypothesis:

$H_{1}: S[n]=h \cdot P[n]+U[n]: P U$ is present

$S[n]$ is sensed signal by SU. $P[n]$ represents the primary signal. $U[n]$ represents noise added by the wireless channel. Here, $n=1,2,3 \ldots . . M$, where $\mathrm{M}$ is the number of samples and $\mathrm{h}$ is the channel gain. It is equal to zero in case of null hypothesis $H_{0}$ and one for alternate hypothesis $H_{1}$. The sensed signal is assumed independent and identically distributed (i.i.d.) random process with mean as zero and variance as given by $E\left[|P(n)|^{2}\right]=\sigma_{p}{ }^{2}$. The noise is assumed as random Gaussian i.i.d. process with zero mean and variance value of $E\left[|U(n)|^{2}\right]=\sigma_{u}{ }^{2}$.

A threshold $(\gamma)$ is defined as per the specification of grade of services. If the test value of $Y[n]$ is greater than threshold then, alternate hypothesis is called, else null hypothesis is called.

For the alternate hypothesis, the received SNR of primary user is given by:

$S N R=\frac{|h|^{2} \sigma_{p}^{2}}{\sigma_{u}^{2}}$

This is based on the assumption that $h, \sigma_{p}{ }^{2}$ and $\sigma_{u}{ }^{2}$ are independent.

\section{Performance metrics}

Energy detector is the most commonly used commercial sensing method. The decision is made by comparing the output of signal detector with a fixed threshold value which helps in deciding the presence or absence of PU (Yücek et al., 2009). The energy detector works on the principle of accumulating the input samples $S[n]$ for estimating the power. It gives an output Y, which acts as a decision statistic (Rashid et al., 2015).

$\mathrm{Y}=\frac{1}{M} \sum_{n=1}^{M} S[n]^{2}$

As the energy detector performs well in low SNR regions, the channel is assumed constant all throughout. Further, the samples are taken to be sufficiently large so that the decision matrix can be approximated using Gaussian distribution.

Sensing accuracy is determined by region of convergence (ROC) curves. These curves are the plots of probability of false alarm with probability of accurate detection or plots of probability of miss detection with probability of false alarm. Probability of detection $\left(\mathrm{P}_{\mathrm{d}}\right)$ is the probability that PU is present and the secondary user is able to detect it. A miss in detecting the licensed user will cause interference between the cognitive device and the primary signal. Probability of false alarm $\left(\mathrm{P}_{\mathrm{f}}\right)$ is probability with which the cognitive device alarms the presence of primary signal even if it is absent. In terms of complementary distribution function of standard Gaussian signal $\mathrm{Q}(),. \mathrm{P}_{\mathrm{d}}$ and $\mathrm{P}_{\mathrm{f}}$ are defined as:

$\mathrm{P}_{\mathrm{f}}=\mathrm{Q}\left(\frac{\gamma-\mu_{0}}{\sigma_{0}^{2}}\right)$

$\mathrm{P}_{\mathrm{d}}=\mathrm{Q}\left(\frac{r-\mu_{1}}{\sigma_{1}^{2}}\right)$ 
Under null hypothesis, probability distribution function (PDF) of Y is approximated as:

mean and variance $\sigma_{0}^{2}=\frac{1}{\sqrt{M}} \sqrt{E|U(n)|^{4}-\sigma_{u}^{4}}$.

In this research, it is assumed that PU signal is complex valued binary phase shift keying (BPSK) signal having least BER and is affected by a circularly symmetric complex Gaussian (CSCG) noise. Here, E $|\mathrm{U}(\mathrm{n})|^{4}=2 \sigma_{\mathrm{u}}^{4}$. Therefore, $\sigma_{0}^{2}=\frac{1}{\sqrt{M}} \sigma_{u}^{2}$. Under the alternate hypothesis, the PDF of $\mathrm{Y}$ is approximated as:

mean $\mu_{1}=(\mathrm{SNR}+1) \sigma_{\mathrm{u}}{ }^{2}$ and variance $\sigma_{1}{ }^{2}=\left\{\mathrm{E}|\mathrm{P}(\mathrm{n})|^{4}+\right.$ $\left.\mathrm{E}|\mathrm{U}(\mathrm{n})|^{4}-\left(\sigma_{\mathrm{P}}^{2}-\sigma_{\mathrm{U}}^{2}\right)^{2}\right\}^{1 / 2}$

As $\mathrm{E}|\mathrm{P}(\mathrm{n})|^{4}=\sigma_{\mathrm{P}}{ }^{4}$ so $\sigma_{1}{ }^{2}=\frac{1}{\sqrt{M}} \sqrt{2 S N R+1} \sigma_{u}^{2}$.

Therefore, the probability pair $\mathrm{P}_{\mathrm{f}}$ and $\mathrm{P}_{\mathrm{d}}$ can be re-written as:

$$
\begin{aligned}
& \left.\mathrm{P}_{\mathrm{f}}=\mathrm{Q}\left(\left(\frac{\Upsilon}{\sigma_{\mu}^{2}}-1\right) \sqrt{M}\right)\right) \\
& \mathrm{P}_{\mathrm{d}}=\mathrm{Q}\left(\frac{\Upsilon}{\sigma_{u}^{2}}-1-S N R\right) \sqrt{\frac{M}{2 S N R+1}}
\end{aligned}
$$

From here, the number of samples $\mathrm{M}$ is derived to be:

$$
\mathrm{M}=\frac{1}{S N R^{2}}\left[\mathrm{Q}^{-1}\left(\mathrm{P}_{\mathrm{f}}\right)-\mathrm{Q}^{-1}\left(\mathrm{P}_{\mathrm{d}}\right) \sqrt{2 S N R+1}\right]^{2}
$$

The theoretical aspect of this mathematics signifies that a threshold value is obtained to achieve a target probability of false alarm under null hypothesis. This threshold is then applied to alternate hypothesis to evaluate probability of detection.

\section{Sensing time-throughput trade-off}

If the sampling time is $\mathrm{T}_{\text {sam }}$, then for $\mathrm{M}$ samples, the sensing time is $T_{s}=T_{\text {sam }} \times$ M. For more number of samples the sensing time is more. If the duration to sense is long, then probability of detection is better. This helps the SUs to access the spectrum holes efficiently, thereby leading to increased throughput. The throughput $(\mathrm{R})$ of the system can be analysed under two cases: Firstly, when primary signal is absent and no false alarm is generated $\left(\mathrm{R}_{0}\right)$; and secondly, when PU is present but SU does not detect it $\left(\mathrm{R}_{1}\right)$.

For the first case: $\mathrm{R}_{0}\left(\mathrm{~T}_{\mathrm{s}}\right)=\frac{T_{f}-T_{s}}{T_{f}}\left(1-\mathrm{P}_{\mathrm{f}}\right) C_{0}^{\prime}$

For the second case: $\mathrm{R}_{1}\left(\mathrm{~T}_{\mathrm{s}}\right)=\frac{T_{f}-T_{s}}{T_{f}}\left(1-\mathrm{P}_{\mathrm{d}}\right) C_{1}^{\prime}$
$C_{0}^{\prime}$ and $C_{1}^{\prime}$ are throughput of secondary signals operating in the absence of primary signal and in the presence of primary signal, respectively. Further,

$$
C_{0}^{\prime}=\log _{2}\left(1+S N R_{S U}\right)
$$

$C_{1}^{\prime}=\log _{2}\left(1+\frac{S N R_{S U}}{S N R_{P U}}\right)$

$\mathrm{SNR}_{\mathrm{SU}}$ is $\mathrm{SNR}$ received of cognitive user at the SU receiver and $\mathrm{SNR}_{\mathrm{PU}}$ is $\mathrm{SNR}$ of licensed user received at the same receiver. The latter being less than one, gives a negative decibel value of SNR. This means that $C_{0}^{\prime}$ is greater than $C_{1}^{\prime}$. The average achievable throughput of the system is given by:

$$
\mathrm{R}\left(\mathrm{T}_{\mathrm{s}}\right)=\mathrm{P}\left(H_{0}\right) \mathrm{R}_{0}\left(\mathrm{~T}_{\mathrm{s}}\right)+\mathrm{P}\left(H_{1}\right) \mathrm{R}_{1}\left(\mathrm{~T}_{\mathrm{s}}\right)
$$

where $\mathrm{P}\left(H_{1}\right)=1-\mathrm{P}\left(H_{0}\right)$ is the probability of presence of PU in the sensed channel. The objective of sensingthroughput problem is to find an optimal sensing time that will give maximum throughput. The objective function can be re-written as:

$\max _{T s} \mathrm{P}\left(H_{0}\right) \mathrm{R}_{0}\left(\mathrm{~T}_{\mathrm{s}}\right)+\mathrm{P}\left(H_{1}\right) \mathrm{R}_{1}\left(\mathrm{~T}_{\mathrm{s}}\right)$

According to Federal Communications Commission (FCC) guidelines the probability of detection should be at least $90 \%$ (Cabric et al., 2004). If the probability of presence of PU is considered less than $20 \%$ (i.e. if $\left.\mathrm{P}\left(H_{1}\right)<0.2\right)$, then $\mathrm{P}\left(H_{0}\right)>0.8$. In addition, as $C_{0}^{\prime}>C_{1}^{\prime}$, the second term of (Equation 15) is considered as negligible as compared to the first term. The objective function can be re-framed as:

$$
\max _{T s} \mathrm{P}\left(H_{0}\right) \frac{T_{f}-T_{s}}{T_{f}}\left(1-\mathrm{P}_{\mathrm{f}}\right) \mathrm{C}_{0}
$$

Furthermore, using the expression for probability of false alarm, throughput is expressed as:

$$
\left.\mathrm{R}\left(\mathrm{T}_{\mathrm{s}}\right)=\mathrm{P}\left(H_{0}\right) \frac{T_{f}-T_{s}}{T_{f}} \mathrm{C}_{0}\left[1-\mathrm{Q}\left(\frac{\gamma}{\sigma_{\mu}^{2}}-1\right) \sqrt{M}\right)\right]
$$

Let $\alpha=\mathrm{Q}^{-1}\left(\mathrm{P}_{\mathrm{d}}\right) \sqrt{2 S N R+1}$

$\mathrm{R}\left(\mathrm{T}_{\mathrm{s}}\right)$ can be written as:

$\mathrm{R}\left(\mathrm{T}_{\mathrm{s}}\right)=\mathrm{P}\left(H_{0}\right) \frac{T_{f}-T_{s}}{T_{f}} \mathrm{C}_{0}\left[1-\mathrm{Q}\left(\alpha+\mathrm{SNR} \sqrt{\frac{T_{s}}{T_{s a m}}}\right)\right]$

This shows that achievable throughput of a cognitive system is a function of sensing time. The spectrum sensing in cognitive radio networks is composed of three major parameters: Sensing time, throughput, and probability of false alarm. 


\section{Fitness function}

The fitness function for optimal sensing is to minimise the sensing time and probability of false alarm and maximise throughput when the frame-period is fixed and the constraint on target probability of detection of at least $90 \%$ is fulfilled. Using the definition of the complementary distribution function, and substituting $\mathrm{a}=\alpha+\mathrm{SNR} \sqrt{\frac{T_{s}}{T_{\text {sam }}}}$, equation (18) can be rearranged as:

$$
\begin{gathered}
\mathrm{R}\left(\mathrm{T}_{\mathrm{s}}\right)=\mathrm{C}_{0} \mathrm{P}\left(H_{0}\right)\left\{\frac{-1}{T_{f}}+\frac{1}{T_{f}} Q\left(\alpha+\sqrt{\frac{T_{S}}{T_{\text {sam }}}} \mathrm{SNR}\right)\right\}+ \\
\mathrm{C}_{0} \mathrm{P}\left(H_{0}\right) \frac{\boldsymbol{T}_{f}-\boldsymbol{T}_{\boldsymbol{s}}}{\boldsymbol{T}_{\boldsymbol{f}}} \frac{\boldsymbol{s N R} \sqrt{\boldsymbol{T S a m}}}{2 \sqrt{2 \pi} \sqrt{\boldsymbol{T} \boldsymbol{s}}} \exp \left[-\frac{\left(\boldsymbol{\alpha}+\mathrm{SNR} \sqrt{\boldsymbol{T}_{s}}\right)^{2}}{2 \sqrt{\boldsymbol{T}_{\text {sam }}}}\right]
\end{gathered}
$$

As $\mathrm{T}_{\mathrm{s}} \rightarrow \mathrm{T}_{\mathrm{f}}$; the second term becomes zero. First term is of the form $\exp \left(-\mathrm{x}^{2} / 2\right)$; i.e. it is a decreasing function, which is always less than 1 , with maximum at 1. Therefore, $\lim _{T s \rightarrow T f} R(T s)<0$

As Ts $\rightarrow 0$; the throughput tends to be infinity as $\mathrm{T}_{\mathrm{s}}$ is in denominator. Therefore, $\lim R(T s)=+\infty>0$. This shows that throughput is a unimodal function having a maxima in the interval $\left(0, \mathrm{~T}_{\mathrm{f}}\right)$. The optimisation function targeting sensing time and throughput can be formulated as in equation (20). The constraints of $90 \%$ probability of detection and fixed frame period are as per FCC guidelines. The limit on sensing time is defined by the maximum time required to sense by a non-optimal system.

$$
\begin{aligned}
& \operatorname{argmax}_{T s}\left(1-\frac{T_{s}}{T_{f}}\right)\left(1-\mathrm{P}_{\mathrm{f}}\right) \\
& \text { subject to }\left\{\begin{array}{c}
T_{s} \leq 31.59 \mathrm{~ms} \\
T_{f}=224.25 \mathrm{~ms} \\
P_{f} \in(0,1]
\end{array}\right.
\end{aligned}
$$

where $\mathrm{T}_{\text {sam }}=0.9 ; \operatorname{SNR} \in(0,1]$;

$$
\mathrm{T}_{\mathrm{S}}=\frac{0.9}{S N R^{2}}\left[\mathrm{Q}^{-1}\left(\mathrm{P}_{\mathrm{f}}\right)-\mathrm{Q}^{-1}\left(\mathrm{P}_{\mathrm{d}}\right) \sqrt{2 S N R+1}\right]^{2}
$$

In this research paper, the problem is investigated by applying the concepts of human opinion dynamics (HOD).

\section{PROPOSED OPTIMISATION ALGORITHM: HUMAN OPINION DYNAMICS}

The human interaction leads to formation of varied opinions in the society. These opinions can be modeled as discrete 'yes' or 'no' or may take continuous values in a given interval. An opinion is a degree of preference of an individual towards a certain fact or thing. It is influenced by other beings as they are diverse in terms of culture, language, religion, beliefs etc. Human beings are social animals and are the most intelligent species. Therefore, the opinion of several human beings can lead to a highly confident and optimised result.

\section{Algorithm framework}

HOD - inspired continuous opinion dynamics optimizer (CODO) is governed by four fundamental pillars, namely, social structure, opinion space, social influence, and updating rule. The framework of the same is depicted in Figure 1.

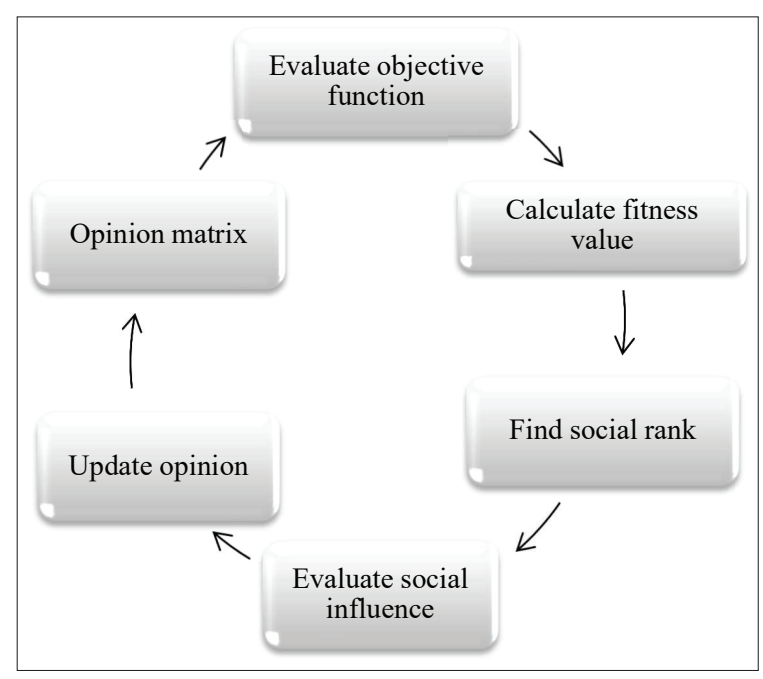

Figure 1: Framework for human opinion dynamic algorithm

Social structure: It is dominated by the interaction amongst the individuals and between them. It takes into consideration the frequency with which they interact, the manner in which they interact. A graph structure is formed wherein an individual is represented by nodes and the links show the neighbourhood set of an individual.

Opinion space: An opinion can be either continuous or discrete. The former can have any real value in the space whereas the latter takes on two values from the set $[0,1]$ or $[-1,1]$. An opinion vector $\mathrm{O}_{i}[\mathrm{t}]$ is formed, which is characterised by opinion of each individual $i$.

Social influence: In a society, the decision making process is based on either one's own decision or on the 
opinion of others. The opinions are formed by interaction, cultural influence, or mass media. An objective function is formulated by considering distance between two individuals and by social ranking of an individual. The social ranks (SR) are governed by the fitness function, which assigns a fitness value to each individual. The objective of the fitness function is to maximise/minimise this value. Lower the value, higher is the rank and vice versa. The individuals having the same value are assigned the same rank. SR is assigned by using the following expression:

$\mathrm{SR}_{\mathrm{j}}(\mathrm{t})=\mathrm{w}_{\mathrm{ij}}(\mathrm{t}) \cdot \mathrm{d}_{\mathrm{ij}}(\mathrm{t})$

Here $\mathrm{SR}_{\mathrm{j}}$ represents social rank of an individual $\mathrm{j} ; \mathrm{w}_{\mathrm{ij}}$ is weight of social influence of $\mathrm{j}$ on $\mathrm{i}$; and $\mathrm{d}_{\mathrm{ij}}$ is Euclidean distance between $i$ and $j$.

Updating rule: The change of opinion is encompassed under updating rule. Any update in opinion leads to change of fitness value and thus the change in rank of an individual in the space. The update rule is governed by the following equation:

$\Delta O_{i}=\frac{\sum_{j=1}^{N}\left(o_{j}(t)-O_{i}(t)\right) w_{i j}(t)}{\sum_{j=1}^{N} w_{i j}(t)}+\xi_{i}(\mathrm{t})$

Here $\mathrm{O}_{i}$ is the opinion of neighbours of $\mathrm{i}$; $\mathrm{N}$ is number of neighbors. $\xi_{i}(t)$ is a normally distributed random noise with mean zero and standard deviation $\sigma_{i}(t)$. This opinion noise is added to avoid early convergence.

$$
\sigma_{\mathrm{i}}(\mathrm{t})=\mathrm{S} \sum_{j=1}^{N} e^{-f i j(t)} \mathrm{p}
$$

$\mathrm{S}$ represents the divergence i.e. it denotes the amount of disintegrating forces. The aim is to converge. The random value is denoted by Gaussian curve. ' $\mathrm{f}$ ' is the fitness value.

\section{CODO: Proposed for spectrum sensing in cognitive radio network}

In this research work, it has been proposed that human opinion dynamics can be applied in cognitive radio networks for spectrum sensing by using CODO technique.

Like the interaction amongst individual human beings, interaction of SU-PU is taken into consideration. The opinion matrix is a collection of randomly generated probability of false alarm values. The values greater/ less than a certain threshold value are considered as presence/ absence of licensed signal and are recorded in the opinion matrix. A fitness function is formulated to optimise the sensing time and the throughput as described in the earlier sections. The fitness value determines the social rank. If there is any change in the decision regarding the presence or absence of PU, then it needs to be updated. An update in the opinion leads to change of fitness value and thus causes a change in the rank. The opinion vector is updated after each iteration. The process is repeated until a preset fitness error value is obtained or maximum number of iterations is performed. A maximum value of iteration is chosen in such a way that the desirable fitness error value can be obtained much earlier than the maximum number of iterations. The algorithm for the HOD as applied to spectrum sensing process is as follows:

Step1: Initialise decision of each SU with some random value in the interval (Xmin, Xmax). The matrix so obtained is named as cognitive.opinion

Step 2: Initialise the iteration to 0

Step 3: Do WHILE (iteration $<$ maximum_value and error $>$ minimum_error)

Step 4: Cognitive.fitness $=$ evaluate_fitness_function (cognitive.opinion); // Using fitness function, calculate fitness values

Step 5: Cognitive.ranking $=$ Calculate_rank (Cognitive. fitness); // Rank each SU based on fitness values

Step 6: iteration ++

Step 7: FOR each individual $\mathrm{i}$ and each dimension $\mathrm{d}$ DO

Step 8: Calculate weight $\left(\mathrm{w}_{\mathrm{ij}}\right)$ of social influence of neighbours $\mathrm{j}$ on $\mathrm{SU} i$.

Step 9: Update decision of individual $\mathrm{i}$

Step 10: Go to Step 3

\section{RESULTS AND DISCUSSION}

In this paper, we have proposed a human opinion dynamics - based CODO algorithm for evaluating the objective function of spectrum-sensing stage of CRs. This section presents the results, which aid in testing the efficacy of the proposed HOD-CODO algorithm for spectrum sensing. The parameters that have been considered for the same are probability of false alarm, probability of detection, throughput, sensing time, and SNR for a fixed frame period with a constraint on maximum sensing time and probability of detection of at least 0.9. Further, a comparison has been drawn to show that proposed algorithm gives better results than non-optimal system and fast convergent particle swarm optimisation technique proposed in literature. The optimisation algorithm was implemented using Matlab and the optimal parameters were investigated. 
Table 1 gives a comparison of proposed HOD-CODO with PSO method in terms of probability of false alarm and normalised throughput for a maximum allowable fixed sensing time of $31.59 \mathrm{~ms}$, SNR of $0.6(-2.2 \mathrm{~dB})$ and $95 \%$ probability of detection. The probability of false alarm for PSO and proposed algorithm is $3.125 \%$ and $2.961 \%$, respectively. The, normalised throughput recorded for the two algorithms are 0.9032 and 0.9039 , respectively. This shows that there is a fall of $5.25 \%$ in the probability of false alarm in HOD-CODO. The chances that SU will declare that the PU is present, when it is not actually present, drop in the proposed algorithm. Therefore, the SU keeps utilising the spectrum hole for its data transmission. This further explains a rise of $0.08 \%$ in normalised throughput offered by human opinion technique.

Table 1: Comparison of proposed algorithm with PSO for fixed Ts (=31.59 ms), $\mathrm{SNR}=0.6(-2.2 \mathrm{db}), \mathrm{Pd}=.95$

\begin{tabular}{lccc}
\hline & PSO & $\begin{array}{c}\text { Proposed } \\
\text { HOD-CODO }\end{array}$ & Improvement \\
\hline Probability of false alarm & 0.03125 & 0.02961 & $5.25 \%$ \\
Normalised throughput & 0.9032 & 0.9039 & $0.08 \%$ \\
\hline
\end{tabular}

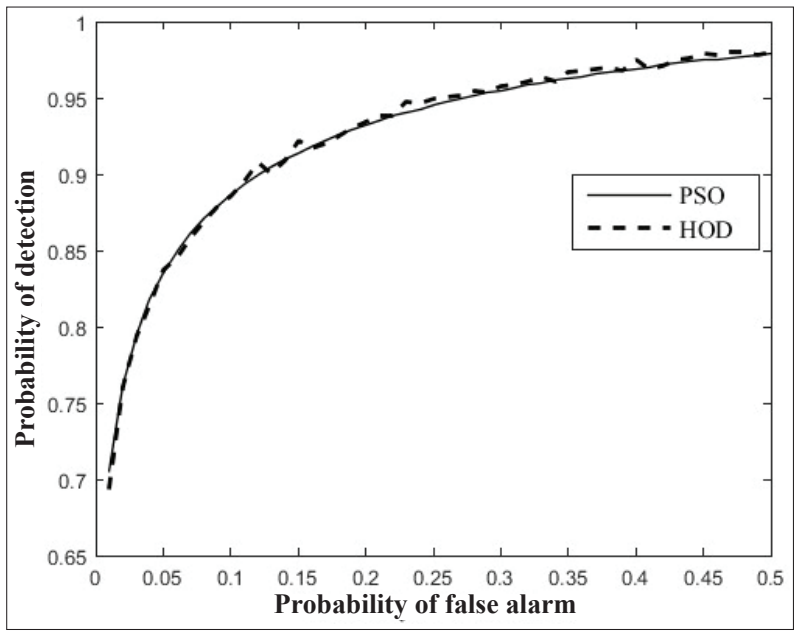

Figure 2: $\quad$ ROC comparison for PSO and HOD-CODO

Figure 2 exhibits the ROC curves for both PSO and HOD-CODO. These illustrate a trend of probability of detection with probability of false alarm. The probability of detection should be maximum while probability of false alarm should be as low as possible. There is a need for negative curve with a steep slope for $\mathrm{P}_{\mathrm{d}}$. However, the graph illustrates that an increase in $P_{d}$ occurs at the cost of increase in $P_{f}$ thereby leading to the best solution state where probability of detection is high for small values of probability of false alarm. The proposed algorithm shows an improvement in the behavior. This assures high protection to the primary user whilst providing sufficient opportunistic access of the radio environment to the

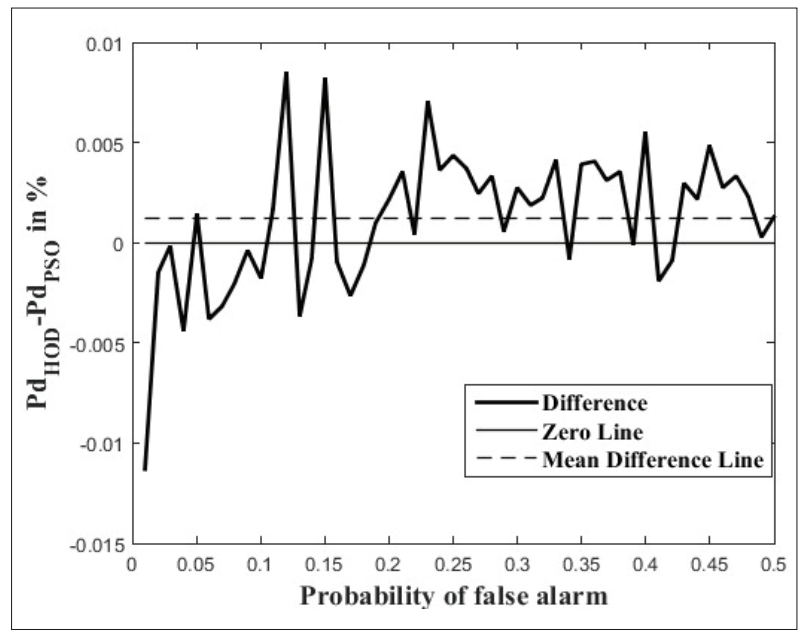

Figure 3: Statistical analysis for probability of detection

cognitive device even though the sensing time has been reduced.

The statistical analysis carried out in Matlab, as illustrated in Figure 3, shows an improvement of $0.2 \%$ in probability of detection by applying the concept of human opinions for spectrum sensing. A good detection assures minimum primary signal interference.

Figure 4 depicts the optimal throughput curves for probability of false alarm and sensing time under varying SNR conditions when the probability of detection has been fixed at the recommended guideline of $90 \%$. The 
rise and the fall of the throughput curve clearly demonstrate the unimodal nature of the function. As the SNR decreases from 1 to 0.6 , the curve shifts towards the lower right side of the graph. This denotes that the maximum value of normalised throughput decreases and sensing time increases. The tip of the curve shows the region of sensingthroughput trade-off. Maximum throughput and minimum sensing time is achieved for SNR of 1 . The sensing time crosses the maximum allowable limit of $31.59 \mathrm{~ms}$ at SNR of 0.6 (-2.2 dB). So, the parameters are evaluated for SNR

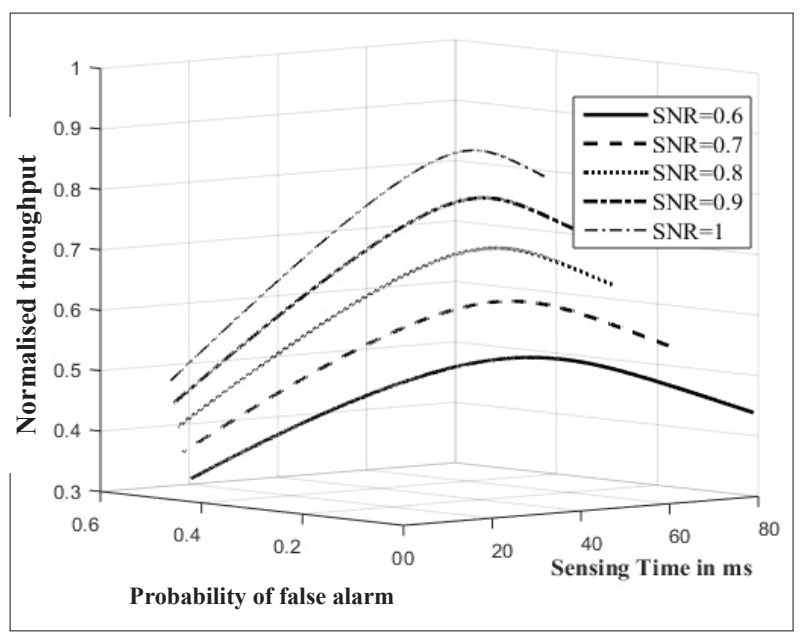

Figure 4: Optimal throughput curve $v s \mathrm{P}_{\mathrm{f}}$ and $\mathrm{T}_{\mathrm{s}}$ under varying SNR at $P_{d}=0.9$

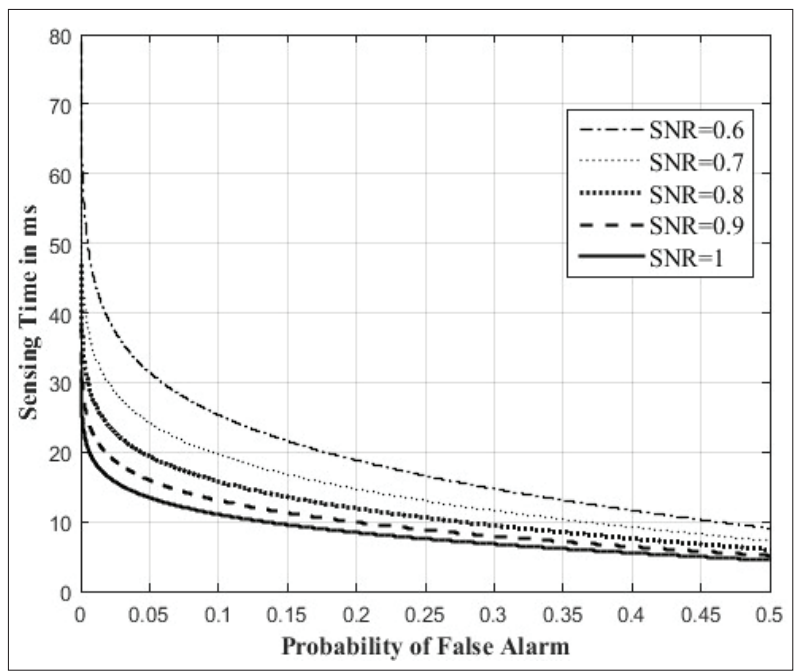

Figure 5: Sensing time $v s$ probability false alarm curve under varying SNR above 0.6. The SNR of 0.6 is denoted as cut-off value, below which cooperative spectrum sensing techniques may be employed for efficient sensing.

Figure 5 elucidates the variation of probability of false alarm with sensing time when SNR is varied from $0 \mathrm{~dB}$ to $-2.2 \mathrm{~dB}$. As the sensing time decreases, there is a rise in false reports. Further, for any sensing time, the probability of false alarm increases with decrease in SNR as the signal strength reduces.

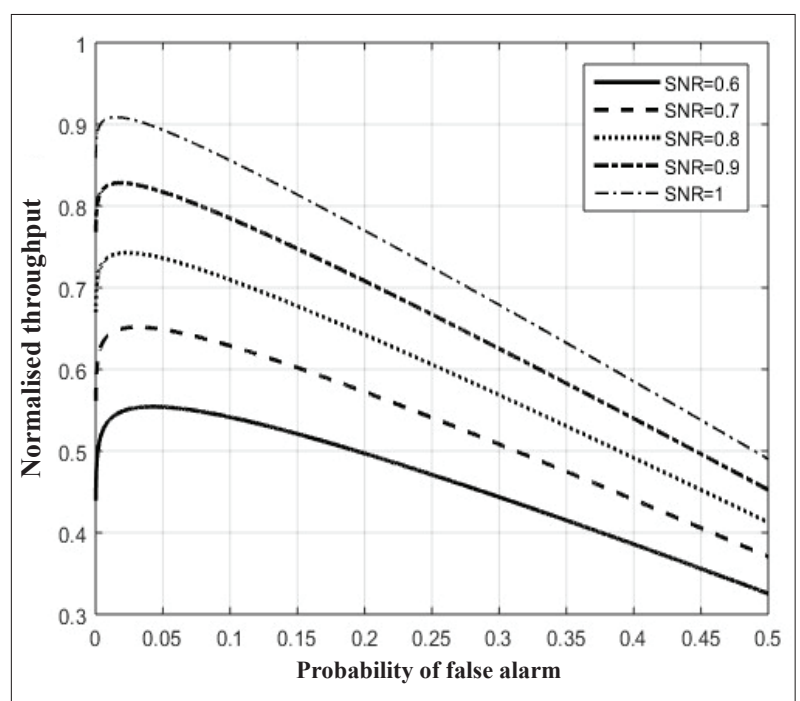

Figure 6: Optimal throughput curve $v s \mathrm{P}_{\mathrm{f}}$ under varying SNR

Table 2: Comparison of non optimal with proposed HOD-CODO for $\mathrm{Ts}=31.59 \mathrm{~ms}$

\begin{tabular}{|c|c|c|}
\hline & $\begin{array}{l}\text { Normalised } \\
\text { throughput }\end{array}$ & $\begin{array}{l}\text { Probability of } \\
\text { false alarm in \% }\end{array}$ \\
\hline Non-optimal system & 0.8161 & 5.0 \\
\hline $\begin{array}{l}\text { Optimal HOD-CODO } \\
\text { technique }\end{array}$ & 0.8610 & 4.0 \\
\hline
\end{tabular}

The fall in throughput with the increase in probability of false alarm is shown in Figure 6 for varying SNR value. As the false reports increase, the throughput falls. This occurs as a result of the SU relinquishing the white spaces due to false alarms generated. The hump shown in the graph is the region of sensing-throughput trade-off. Table 2 compares the normalised throughput and the probability of false alarm for a non-optimal system with proposed algorithm for a fixed sensing time of $31.59 \mathrm{~ms}$. 
The normalised throughput and the probability of false alarm are recorded as 0.8610 and $4 \%$, respectively as compared to 0.8161 and $5 \%$, respectively, for the non- optimal system. This shows an improvement of $5.5 \%$ in normalised throughput and $25 \%$ drop in probability of false alarm.

Table 3: Comparison of optimal PSO, and proposed HOD-CODO when Ts $\leq 31.59 \mathrm{~ms}$

\begin{tabular}{lccc}
\hline & $\begin{array}{c}\text { Sensing time } \\
\text { in ms }\end{array}$ & $\begin{array}{c}\text { Normalised } \\
\text { throughput }\end{array}$ & $\begin{array}{c}\text { Probability of } \\
\text { false alarm in \% }\end{array}$ \\
\hline $\begin{array}{l}\text { Optimal PSO technique } \\
\text { with adaptive Ts }\end{array}$ & 17.3648 & 0.9088 & 1.5 \\
$\begin{array}{l}\text { Optimal HOD-CODO } \\
\text { technique with adaptive Ts }\end{array}$ & 17.100 & 0.9088 & 1.6 \\
\hline
\end{tabular}

Table 3 presents the comparison of optimal PSO with proposed HOD-CODO when the sensing time is varying. The sensing time-throughput values for varying SNR are shown in Figure 4. Least sensing time of $17.10 \mathrm{~ms}$ with a normalised throughput of 0.9088 and probability of false alarm of $1.6 \%$ is recorded at SNR $=1$ for the proposed algorithm. These respective values for PSO are $17.3648 \mathrm{~ms}$ and 0.9088 with probability of false alarm of $1.5 \%$ for the same SNR (Rashid et al., 2015). A comparison of Table 2 and Table 3, shows an improvement of $45 \%$ in sensing time, $11 \%$ in throughput, and $70 \%$ in probability of false alarm as compared to non-optimal system. Further, the proposed human opinion method shows an improvement in sensing time for nearly the same throughput. However, a slight decrease in probability of false alarm is due to the random nature of this scheme. A sensing time of $17.1 \mathrm{~ms}$ suggests that $8 \%$ of the frame is utilised for sensing and $92 \%$ of the frame is employed for data transmission, thus leading to rise in throughput.

The promising results achieved by proposed method for spectrum sensing are attributed to the social behaviour and the intelligence possessed by the human beings. Humans interact and apply their intelligence to make decisions as opposed to the animal behaviour. Furthermore, the decision is based on opinion influenced by the various factors like social, environmental, personal etc. In a crowd, there is a large probability that each being will have a different opinion and an individual will not simply follow others, unlike the herd mentality of birds, fishes, or ants. It is hard to predict ones opinion, which provides randomness to the algorithm. Further, repetitive discussions may lead to change of opinion of an individual. Humans communicate and interact iteratively to reach a final decision, which is high on the confidence level.

\section{CONCLUSION}

This paper proposes human opinion dynamics inspired continuous opinion dynamics optimiser for spectrum sensing in cognitive radio networks. The parameters under consideration are throughput, probability of false alarm, and sensing time under varying signal-to-noise ratio conditions with at least $90 \%$ probability of detection, fixed frame period of $224.25 \mathrm{~ms}$, and maximum sensing time of $31.59 \mathrm{~ms}$.

The proposed method shows a fall of $5.25 \%$ in probability of false alarm and an increase of $0.08 \%$ in the throughput as compared to PSO under the condition that probability of detection is $95 \%$ for fixed frame period of $224.25 \mathrm{~ms}$ with maximum sensing time of $31.59 \mathrm{~ms}$. This implies that the chances drop in HOD of SU declaring that PU is present, when it is actually not present. The comparison of ROC curves of PSO and HOD-CODO depicts that the proposed algorithm gives performance improvement of $0.2 \%$ in probability of detection by employing this scheme under the condition imposed by FCC that the probability of detection is at least $90 \%$. This assures high protection to the PU while satisfying the selfish interest of the SU. As the SNR increases, the throughput increases, the sensing time falls and probability of false alarm drops. A high probability of false reports is recorded for low sensing time. However, low sensing time leads to higher throughput. Therefore, a trade-off of these parameters yields that a maximum normalised throughput of 0.9088 is recorded at minimum sensing time of $17.10 \mathrm{~ms}$. This low sensing time means that only $8 \%$ of the total frame period is utilised for sensing as opposed to $14 \%$ by non-optimal system. Thus, there is a rise in data transmission. Further, the results describe that at the maximum allowable sensing 
time limit of $31.59 \mathrm{~ms}$, the cut-off value for SNR is 0.6 $(-2.2 \mathrm{~dB})$; thereby recommending the use of cooperative spectrum sensing for transmissions at lower SNR values. The encouraging results exhibited by human opinion method are a result of social behavior of intelligent human species. Human beings have a tendency to interact and apply intelligence to make decisions, unlike herd mentality of animals. The future scope of research can be steered towards implementing a hybrid of PSO and HOD for spectrum sensing.

\section{REFERENCES}

Aulakh I.K. \& Vig R. (2015). Secondary user sensing time optimization in sensing-transmission scheduling for dynamic spectrum access in cognitive radio. Journal of Computer Science 11(08): 880-891. DOI: https://doi.org/10.3844/jcssp.2015.880.891

Bkassiny M., Li Y. \& Jayaweera S.K. (2013). A survey on machine-learning techniques in cognitive radios. IEEE Communications Surveys and Tutorials 15(3): 1136-1159. DOI: https://doi.org/10.1109/SURV.2012.100412.00017

Cabric D., Mishra S.M. \& Brodersen R.W. (2004). Implementation issues in spectrum sensing for cognitive radios. Proceedings of the $38^{\text {th }}$ IEEE Asilomar Conference on Signals, Systems and Computers (eds. M.B. Matthews), volume 38, Pacific Grove, USA, 7-10 November. The Naval Postgraduate School, Monterey, California, USA, pp. 772-776.

DOI: https://doi.org/10.1109/ACSSC.2004.1399240

Clancy C., Hecker J., Stuntebeck E. \& O'Shea T. (2007). Applications of machine learning to cognitive radio networks. IEEE Wireless Communications 14(4): 47-52. DOI: https://doi.org/10.1109/MWC.2007.4300983

Deffuant G., Neau D., Amblard F. \& Weisbuch G. (2000). Mixing beliefs among interacting agents. Advances in Complex Systems 3(01): 87-98.

DOI: https://doi.org/10.1142/S0219525900000078

Garg R. \& Saluja N. (2016). Current trends and research challenges in spectrum-sensing for cognitive radios. International Journal of Advanced Computer Science and Applications 7(07): 403-408.

DOI: https://doi.org/10.14569/IJACSA.2016.070756

Giupponi L., Galindo-Serrano A., Blasco P. \& Dohler M. (2010). Docitive networks: An emerging paradigm for dynamic spectrum management [Dynamic Spectrum Management]. IEEE Wireless Communications 17(4): 47-54.

DOI: https://doi.org/10.1109/MWC.2010.5547921

Hegselmann R. \& Krause U. (2005). Opinion dynamics driven by various ways of averaging. Computational Economics 25(4): 381-405.

DOI: https://doi.org/10.1007/s10614-005-6296-3

Jhajj H.K., Garg R. \& Saluja N. (2017). Efficient spectrum sensing in cognitive radio networks using hybridized particle swarm intelligence and ant colony algorithm.
International Journal on Communications Antenna and Propagation 7(07): 586-593.

DOI: https://doi.org/10.15866/irecap.v7i7.12434

Kaur R., Kumar R., Bhondekar A.P. \& Kapur P. (2013). Human opinion dynamics: An inspiration to solve complex optimization problems. Scientific Reports 3: 1-7. DOI: https://doi.org/10.1038/srep03008

Kim H. \& Shin K.G. (2008). In-band spectrum sensing in cognitive radio networks: Energy detection or feature detection? In: MobiCom 2008. Proceedings of the $14^{\text {th }}$ ACM International Conference on Mobile Computing and Networking (eds. J.J. Garcia-Luna-Aceves, R. Sivakumar \& P. Steenkiste), volume 1, San Francisco, California, USA, 14-19 September. pp. 14-25.

DOI: https://doi.org/10.1145/1409944.1409948

Liang Y. C., Zeng Y., Peh E.C.Y. \& Hoang A.T.H. (2008). Sensing-throughput tradeoff for cognitive radio networks. IEEE Transactions on Wireless Communications 7(04): 1326-1337. DOI: https://doi.org/10.1109/TWC.2008.060869

Liu X., Zhong W. \& Chen K. (2015). Optimization of sensing time and cooperative user allocation for OR-rule cooperative spectrum sensing in cognitive radio network. Journal of Central South University 22(7): 2646-2654. DOI: https://doi.org/10.1007/s11771-015-2795-0

Macaš M., Gerla V. \& Lhotská L. (2012). Automatic neonatal sleep EEG recognition with social impact based feature selection. In: Advances in Intelligent Systems and Computing (eds. V. Snášel, A. Abraham \& E. Corchado), pp. 389-398. Springer, Berlin, Heidelberg, Germany. DOI: https://doi.org/10.1007/978-3-642-32922-7_40

Mäs M., Flache A. \& Helbing D. (2010). Individualization as driving force of clustering phenomena in humans. PLoS Computational Biology 6(10):1-9.

DOI: https://doi.org/10.1371/journal.pcbi.1000959

Ozbay S. \& Ercelebi E. (2015). A new wireless network scheme for spectrum sensing in cognitive radio. Elektronika Ir Elektrotechnika 21(6): 90-95. DOI: https://doi.org/10.5755/j01.eie.21.6.13769

Pandya P., Durvesh A. \& Parekh N. (2015). Energy detection based spectrum sensing for cognitive radio network. Proceedings of the $5^{\text {th }}$ IEEE International Conference on Communication Systems and Network Technologies (eds. G. Tomar), volume 5, Gwalior, India, 4-6 April. Machine Intelligence Research Labs, Shriram College of Engineering \& Management, Gwalior, India, pp. 201-206. DOI: https://doi.org/10.1109/CSNT.2015.264

Poddar S., Hussain S., Ailneni S., Kumar V. \& Kumar A. (2016). Tuning of GPS aided attitude estimation using evolutionary algorithms. International Journal of Intelligent Unmanned Systems 4(1): 23-42.

DOI: https://doi.org/10.1108/IJIUS-05-2015-0005

Rashid R.A., Hamid A. H. F. B. A., Fisal N., Kamilah S.-Y., Hosseini H., Lo A. \& Farzamnia A. (2015). Efficient in-band spectrum sensing using swarm intelligence for cognitive radio network. Canadian Journal of Electrical and Computer Engineering 38(2): 106-115. DOI: https://doi.org/10.1109/CJECE.2014.2378258 
Smith P.J., Dmochowski P.A., Suraweera H.A. \& Shafi M. (2012). The effects of limited channel knowledge on cognitive radio system capacity. IEEE Transactions on Vehicular Technology 62(2): 927-933. DOI: https://doi.org/10.1109/TVT.2012.2227864

So J. \& Kwon T. (2016). Limited reporting-based cooperative spectrum sensing for multiband cognitive radio networks. $A E U$ - International Journal of Electronics and Communications 70(4): 386-397.

DOI: https://doi.org/10.1016/j.aeue.2015.12.017

Stotas S. \& Nallanathan A. (2010). Overcoming the sensing-throughput tradeoff in cognitive radio networks. Proceedings of the IEEE International Conference on Communications (eds. F. Takawira \& W. Leuschner), Cape Town, South Africa, 23-27 May. International Convention Centre, Cape Town, South Africa, pp. 3-7. DOI: https://doi.org/10.1109/ICC.2010.5502792

Suseela B. \& Sivakumar D. (2017). Cognitive radio and its impact of throughput with channel optimization techniques. Journal of Computational and Theoretical Nanosciences 14(1): 430-434. DOI: https://doi.org/10.1166/jctn.2017.6339

Thilina K.M., Saquib N. \& Hossain E. (2013). Machine learning techniques for cooperative spectrum sensing in cognitive radio networks. IEEE Journal on Selected Areas in Communications 31(11): 2209-2221. DOI: https://doi.org/10.1109/JSAC.2013.131120

Wickramage C. \& Ranasinghe D.N. (2014). Modelling altruistic and selfish behavioural properties of ant colony optimisation. Proceedings of the International Conference on Advances in ICT for Emerging Regions (eds. D.D. Karunaratne), Colombo, Sri Lanka, 10-13 December. University of Colombo School of Computing, Colombo, Sri Lanka, pp. 85-90.

DOI: https://doi.org/10.1109/ICTER.2014.7083884

Wickramarathne T., Premaratne K. \& Murthi M. (2013). Toward efficient computation of the Dempster-Shafer belief theoretic conditionals. IEEE Transactions on Cybernetics 43(2): 712-724.

DOI: https://doi.org/10.1109/TSMCB.2012.2214771

Xiao S., Li S., Li Z. \& Zhang X. (2014). Improving the throughput of sensing-based spectrum sharing in wideband cognitive radio networks. ICIC Express Letters 8(11): 3157-3163.

$\mathrm{Xu}$ X., Qu R., Zhao J. \& Chen B. (2015). Cooperative spectrum sensing in cognitive radio networks with kernel least mean square. Proceedings of the $5^{\text {th }}$ IEEE International Conference on Information Science and Technology, Changsha, China, 24-26 April. pp. 574-578. DOI: https://doi.org/10.1109/ICIST.2015.7289037

Yücek T. \& Arslan H. (2009). A survey of spectrum sensing algorithms for cognitive radio applications. IEEE Communications Surveys and Tutorials 11(1):116-130. DOI: https://doi.org/10.1109/SURV.2009.090109

Zhang S., Hafid A.S., Zhao H. \& Wang S. (2016). Crosslayer rethink on sensing-throughput tradeoff for multichannel cognitive radio networks. IEEE Transactions on Wireless Communications 15(10): 6883-6897. DOI: https://doi.org/10.1109/TWC.2016.2592959 